\title{
1 The origin and maintenance of microbial symbionts in Drosophila 2 larvae
}

3

4 Robin Guilhot $^{1 *}$, Auxane Lagmairi ${ }^{1}$, Laure Olazcuaga ${ }^{1}$, Anne Xuéreb $^{1}$, Simon Fellous ${ }^{1}$

$5{ }^{1}$ CBGP, INRAE, CIRAD, IRD, Montpellier SupAgro, Univ Montpellier, Montpellier, France

$6 \quad *$ corresponding author's email address: guilhoro@gmail.com

\section{Abstract}

9 Little is known on the origin and maintenance of symbionts associated with Drosophila larvae 10 in natura, which restricts the understanding of Drosophila-extracellular microorganism 11 symbiosis in the light of evolution. Here, we studied the origin and maintenance of symbionts 12 of Drosophila larvae under ecologically realistic conditions, to our knowledge for the first 13 time, using yeast and bacterial isolates and two Drosophila species: the model organism $D$. 14 melanogaster and the invasive pest D. suzukii. We discovered that Drosophila females and 15 males both transmit yeast and bacteria symbionts to larvae. In addition, several symbiotic 16 yeasts initially associated with larvae were conserved throughout host life cycle and 17 transmitted to offspring. Our results suggest that stable associations of Drosophila flies with 18 bacteria and yeasts may exist in natura and constitute a step forward in the understanding of 19 wild Drosophila -microorganism symbioses. 
The origin of microbial symbionts of eukaryotes influences the evolution of symbiosis. Microbial symbionts can be acquired from parents (i.e. vertically transmitted symbionts) (Funkhouser \& Bordenstein 2013), from unrelated hosts (i.e. horizontally transmitted symbionts) (Gonella et al. 2012), a mix of both (i.e. mixed-mode transmitted symbionts) (Ebert 2013; Quigley et al. 2018) or from the environment (Kikuchi et al. 2007). Theory predicts that symbionts that persist between host life stages and host generations are more likely to initiate stable mutualistic relationships compared to symbionts acquired from the host environment (Antonovics et al. 2017; Bright \& Bulgheresi 2010; Fisher et al. 2017; Gerardo \& Hurst 2017; Lipsitch et al. 1996; Sachs et al. 2004; Shapiro \& Turner 2014). Understanding the evolution of host-microbe symbiosis is therefore only possible when means of host-microbe association are properly documented.

In Drosophila flies, numerous studies conducted under laboratory conditions investigated the origin of extracellular microbial symbionts associated with larvae and the persistence of larval symbionts throughout host life cycle (Bakula 1969; Becher et al. 2012; Pais et al. 2018; Téfit et al. 2018). However, little is known on the origin and maintenance of symbionts associated with Drosophila larvae in natura, which restricts the understanding of Drosophilaextracellular microorganism symbiosis in the light of evolution. We explored these phenomena under ecologically realistic conditions, to our knowledge for the first time, using mainly wild - yeast and bacterial isolates and two Drosophila species of major interest: the model organism $D$. melanogaster and the invasive pest $D$. suzukii.

\section{Results and Discussion}

\section{Drosophila females transmit extracellular symbionts to their offspring}

Previous reports showed D. melanogaster maternal transmission of yeasts and bacteria in laboratory conditions (Bakula 1969; Becher et al. 2012; Rohlfs and Hoffmeister 2005; Spencer et al. 1992; Téfit et al. 2018). We hypothesized that Drosophila mothers may transmit their microbial symbionts to larvae in a context where other microorganisms are present on the oviposition substrate. We also predicted that D. suzukii maternal transmission may be more frequent that of $D$. melanogaster because $D$. suzukii females typically lay their 
50

51

52

53

54

55

56

eggs on unwounded, ripening fruits poorly colonized by microorganisms (Lewis \& Hamby 2019). D. suzukii eggs are inserted in fruit flesh thanks to females' serrated ovipositors. As a result, the newly emerged larvae may primarily recruit microbial symbionts deposited by the mother. By contrast, D. melanogaster females lay their eggs on fruit wounds and rotten fruits already colonized by a variety of microorganisms (data not shown, will be available in the next version of this work). To test these predictions, we used mature females of one D. suzukii population and one D. melanogaster population and six microbial symbionts (see Materials and Methods for details on their choice). The same microbial strains were used for all the experiments presented in our study. Briefly, individual mated female associated with an artificial microbial community composed of one bacterium and one yeast strain were offered to oviposit on a blueberry which surface had been inoculated with a different microbial community (i.e. another bacterium and another yeast) (Figure 1A). For D. melanogaster assays the berry was slightly wounded while kept unwounded for D. suzukii assays. Five days after fruit exposure, numerous berries contained larvae associated with female microbial symbionts, fruit-surface microorganism or both (Figure 1B).

Contrary to our expectations, maternal transmission was no greater in $D$. suzukii than in $D$. melanogaster. However, symbiont identity affected both maternal transmission and environmental acquisition (Table S1). One yeast strain, Trigonopsis vinaria isolated from $D$. suzukii ovaries was transmitted significantly more from $D$. melanogaster than from $D$. suzukii females $\left(\chi^{2}=5.25, \mathrm{df}=1, \mathrm{p}=0.0220\right.$ ) (Figure $1 \mathrm{C}$, Table S1). Symbiont transmission differed whether they were in females or on fruit, which suggests acquisition of maternal symbionts by offspring is controlled by interactions between females and symbionts rather than symbiont's sheer properties. Our work indicates D. suzukii and D. melanogaster maternal transmission of extracellular symbionts may be frequent in field conditions. 
A

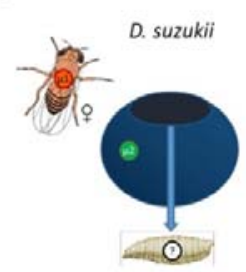

B
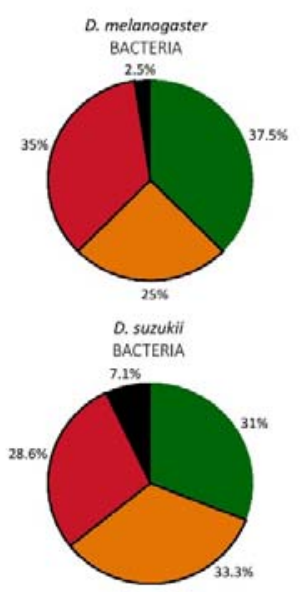
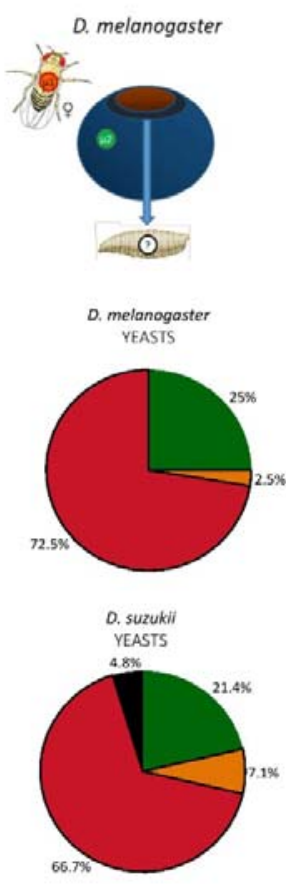

C
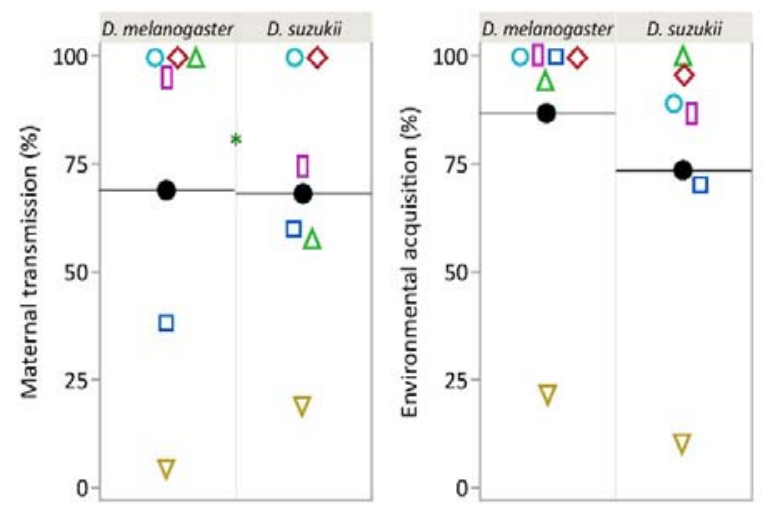

Origin of larval-associated symbionts (\%)

Microbial symbionts

Yeasts Bacteria

$\square$ R. babjevae $O G$. thailandicus

$\checkmark$ H. uvarum IS. liquefociens

$\Delta T$. vinaria $\nabla$ L.plantarum

Figure 1. Drosophila larvae associate with maternal symbionts and environmental symbionts. (A) Experimental design. Three different microbial communities $\left(\mu_{i}\right)$ composed of a yeast and a bacterium species were permuted between flies and fruits. $n=40 D$. melanogaster experimental units; $n=42$ D. suzukii experimental units. (B) Drosophila larvae frequently harbored maternal symbionts and those already present on fruit skin. (C) Maternal transmission and environmental acquisition rates (\% of larvae pools). The black dot symbolizes the general mean (i.e. independently of the microbial symbiont) and the open symbols the proportion for each of the six microorganisms tested.

\section{Male transmission of microbial symbionts}

How microorganisms reach fruit skin, where they are recruited by Drosophila larvae, is unclear. Insects, such as wasps, participate to baker's yeast spread at the landscape level (Stefanini et al. 2012). Field observations showed Drosophila males often sit on fruit, a behavior that we also witnessed in lab macrocosms (SM2). We hence hypothesized Drosophila males may deposit their symbionts on fruit surface and therefore contribute to the larval microbiota. D. melanogaster males can be territorial, can form leks and defend oviposition sites (Drapeau et al. 2011; Hoffmann and Cacoyianni 1990). Because D. melanogaster males are present on fruit wounds (i.e. oviposition sites), where microorganisms 
could grow better than on fruit skin, we predicted greater male transmission from $D$. melanogaster than from D. suzukii. In a new experiment we tested whether Drosophila males actually transmitted their microbial symbionts to offspring of conspecific females (Figure 2A). Individual males were given single blueberries for $24 \mathrm{~h}$ until single females were added for another $24 \mathrm{~h}$. As before, males, females and fruits were all associated with different microbial communities.

Male transmission to larvae was pervasive and twice more frequent for D. melanogaster (c. $50 \%$ of fruits) than D. suzukii (c. 25\%) (Figures 2B and 2C). The transmission by males of the microorganisms widely depended on strain identity. For example, the yeast $H$. uvarum was always transmitted by $D$. melanogaster males while the yeast $R$. babjevae was never found in larvae. Female transmission was slightly lower than that of in the first experiment, with different behaviors of the microbial strains (Figure 2C, Table S3). The transmission potential of the symbiont strains appeared different in males and females and among experiments in females suggesting this aspect of strain biology is very context-sensitive.

How did males transmit their symbionts? We recorded the time they spent on oviposition areas but this variable did not correlate significantly with the transmission of their symbionts to larvae (Table S3). Male transmission is therefore not determined by the amount of microbial cells they shed on oviposition sites. Similarly, we recorded whether males and females mated during the experiment. These events were rare $(\mathrm{n}=7 / 21$ observations for $D$. melanogaster and $\mathrm{n}=2 / 27$ for $D$. suzukii) and did not influence significantly male transmission (Table S3). This suggests that male transmission of symbionts to larvae did not depend from male presence on oviposition sites and did not clearly involve sexual transmission to females (Miest and Bloch-Qazi 2008; Rohlfs and Hoffmeister 2005; Starmer, Peris and Fontdevila 1988). Independent of the mechanisms, the transmission of symbionts by Drosophila males may have consequences for the evolution of symbionts effects on males. Microorganisms may change male characters so as to favor their transmission. Because symbiont transmission is not contingent upon male reproduction (i.e. no male vertical transmission), selection may not select against symbiont costs to male fitness (Sachs 2004; Ebert 2013). However, the largest D. melanogaster males would be most likely to successfully defend oviposition sites (Hoffmann 1987). Therefore, symbionts of male larvae would be selected for beneficial effects on their development, assuming that extracellular symbionts of larvae remain associated with their hosts after metamorphosis and until they reproduce. 
A

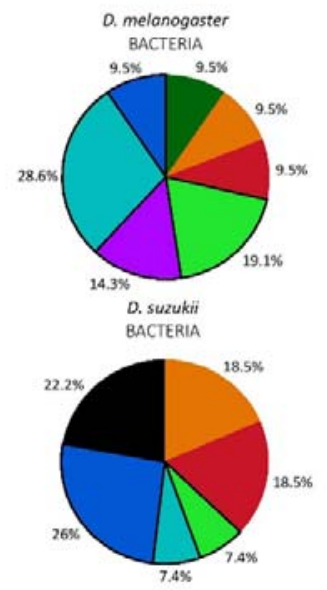

Origin of larval-associated symbionts (\%)

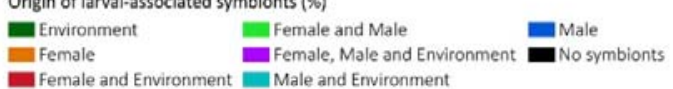

D. melanogaster YEASTS
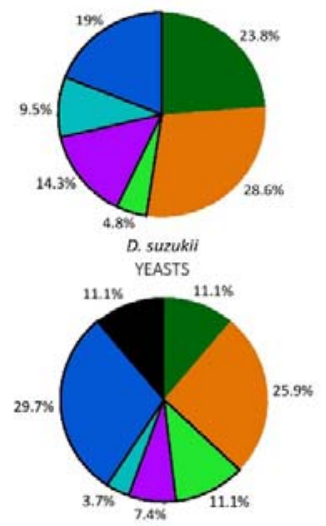

and Male
D. melanogaster

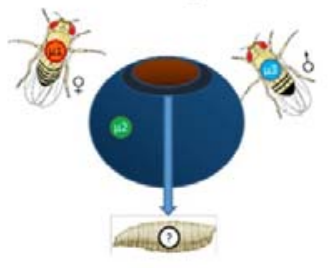

Figure 2. Drosophila larvae associate with male symbionts, maternal symbionts and
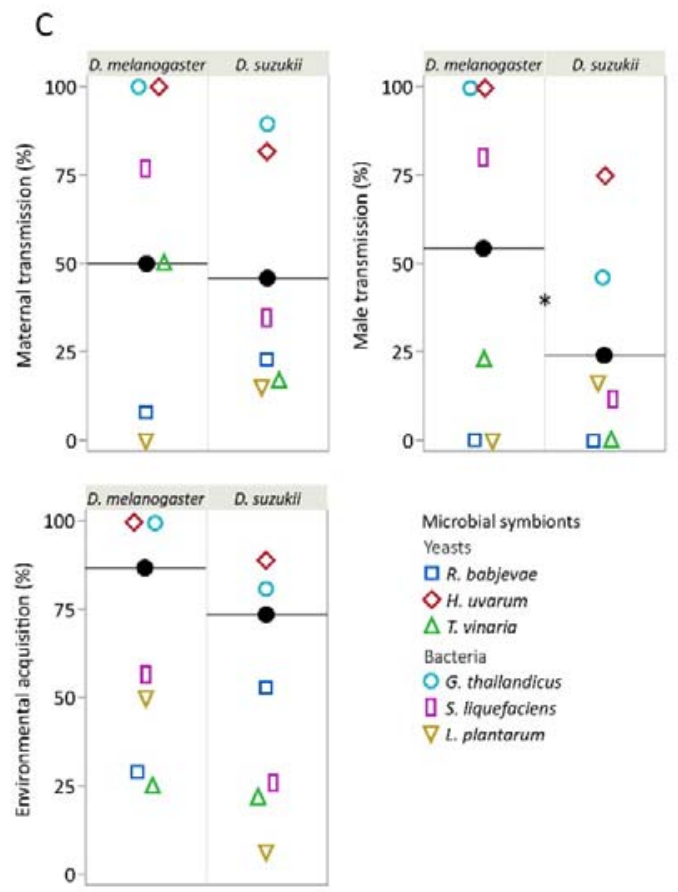

Microbial symbionts

Yeasts

ㅁ. babjevae

H. uvarum

$\Delta \tau$. vinaria

Bacteria

प S. liquefaciens

$\nabla$ L. plantarum
O G. thallandicus environmental symbionts. (A) Experimental design. Three different microbial communities $\left(\mu_{i}\right)$ composed of a yeast and a bacterium species were permuted between flies and fruit. $n=$ 21 D. melanogaster experimental units; $n=27$ D. suzukii experimental units. (B) Drosophila larvae frequently harbored symbionts of both male and female as well as those already present on fruit skin. (C) Proportion of cases where larvae contained male, female and fruit symbionts (\% of larval pools). The black dot symbolizes the general mean (i.e. independently of the microbial symbiont) and the open symbols the proportion for each of the 6 microorganisms tested.

\section{Larval yeast symbionts maintain through the entire life cycle and transmit}

\section{to the progeny}

Do extracellular symbionts of Drosophila larvae maintain until adult life? Several studies have shown symbionts of larvae can be found in adults (i.e. transstadial transmission, maintenance through metamorphosis) (Bakula 1969; Duneau and Lazzaro 2018; Ridley et al. 2012; Starmer, Peris and Fontdevila 1988). However, in most experiments larvae and adults shared the same containers hence permitting indirect, environmental transmission (but see Bakula 1969). In the field, Drosophila last-instar larvae mainly pupate outside the larval 
environment, usually in soil (Reaume \& Sokolowski 2006; Woltz \& Lee 2017). This behavior was mimicked in a new experiment where Drosophila larvae were associated with one of three yeast strains, newly formed pupae isolated in independent containers and adult microbial content assayed shortly after emergence (Figure 3A).

Almost a quarter and a half of young D. suzukii and D. melanogaster adults tested positive for larval symbionts (cell numbers where however usually low). Trigonopsis vinaria yeast, isolated from D. suzukii ovaries, best maintained through host metamorphosis while our strain of Hanseniaspora uvarum, a species frequently found in wild Drosophilids, exhibited poor transstadial transmission $\left(\chi^{2}=0.0188, \mathrm{df}=2, \mathrm{p}=0.0188\right.$ ) (Figure 3B). Fly species and adult sex had marginally non-significant influences on yeast transstadial maintenance (for both, $\chi^{2}=$ 3.70 , df $=1, p=0.0544$ ) (Figure 3B). Overall, yeast symbionts of larvae maintained until adult emergence, but what would become of them remained to be determined.

Numerous laboratory experiments indicate adult symbiotic community mirrors that of their surrounding environment due to the constant replacement of gut microbial communities during feeding (Blum et al. 2013; Ma \& Leulier 2018). However, other work shows adult association with some nutritional symbionts, in particular those recently isolated from the field, may be stable (Pais et al. 2018; Obadia et al. 2017). We continued the previous experiment in order to determine whether yeast present in young adults, and acquired at the larval stage, maintained through life and until the next generation (Figure 3A). Freshly emerged adults associated with one of three yeast strains were maintained for five days with a halved grape berry inoculated with a second yeast strain (i.e. first environmental symbiont in Figure 3A) and another two days with berries inoculated with a third strain (i.e. third environmental symbiont). These adults were then offered a surface-sterilized berry to oviposit. The microbial content of adults at the time of oviposition and that of F1 larvae was assayed.

Unexpectedly, yeast symbionts of larval origin largely maintained despite a one-week exposure to two successive sources of environmental yeasts (Figure 3C, Table S4). The second environmental yeast was less frequent in adults compared to the first (Figure 3C). Even if the time of adult exposure to symbionts may affects host acquisition (as in Obadia et al. 2017), it should be mentioned we did not monitor microbial development in the grape berries the flies were exposed to for five and two days, respectively. It is therefore possible flies inoculated them with the strains they harbored, hence favoring the multiplication of larval and first environmental symbionts in our microcosms and their subsequent re- 
178 inoculation to adults. Incidentally, it could explain the greater prevalence of larval yeast in old 179 adults than in young adults (compare Figures 3B and 3C). Nonetheless, the majority of $D$. 180 melanogaster larvae of the following generation bore symbionts their parents were first 181 exposed to at the larval stage (proportion of larvae with the larval symbionts of their parents: 1820.69 (95\% CI $[0.44,0.86])$ ) (Figure 3D). This experiment shows that, in field-realistic 183 conditions, symbiotic yeasts associated with D. melanogaster and D. suzukii larvae are 184 conserved in young adults despite metamorphosis and illustrate symbiont persistence 185 throughout host life cycle until they are transmitted to a new generation. 
A

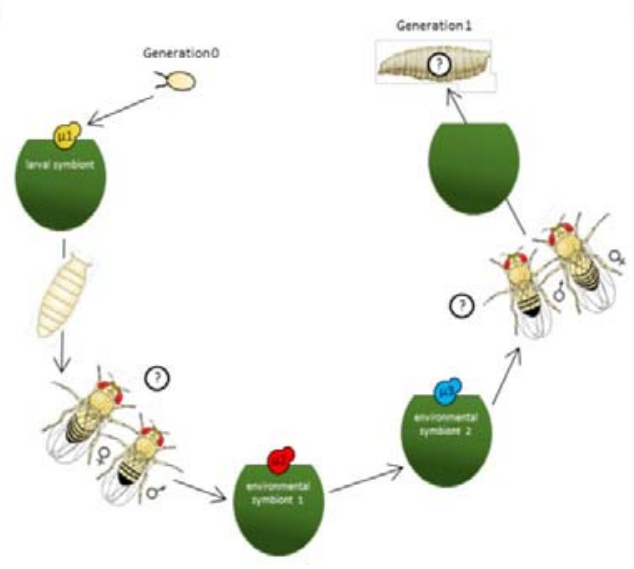

B

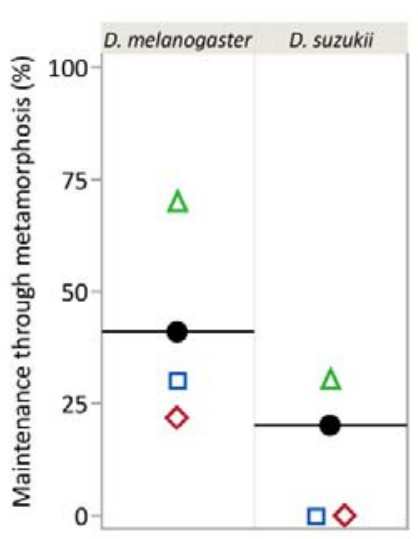

Yeast symbionts

$\square$ R. babjevae

$\diamond$ H. uvarum

$\triangle T$. vinaria

C
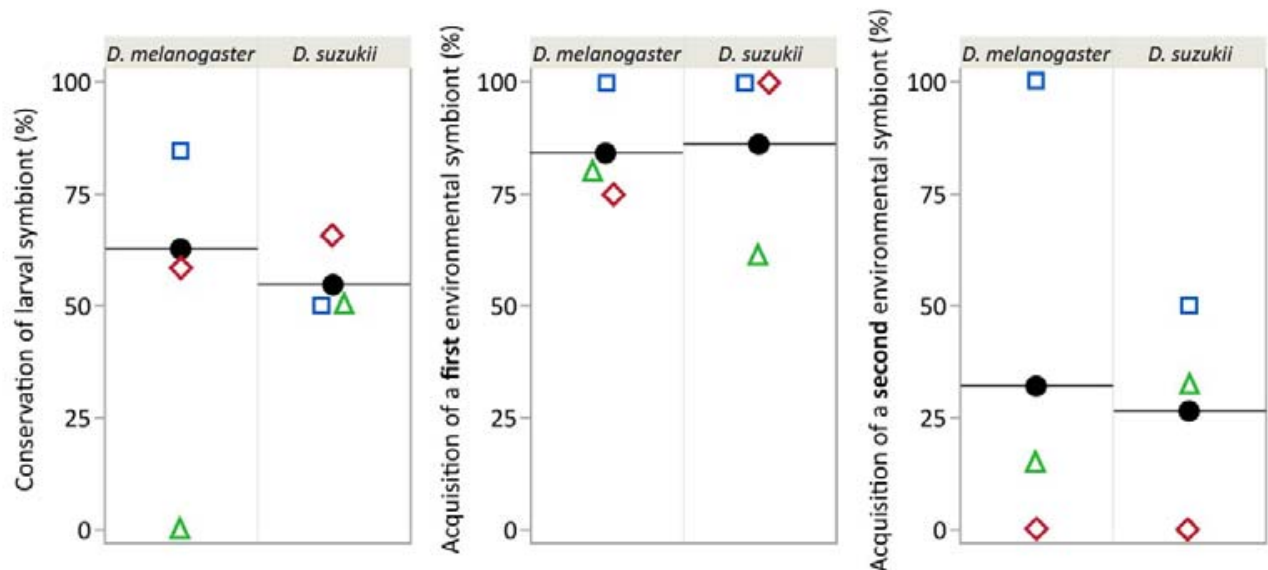

D
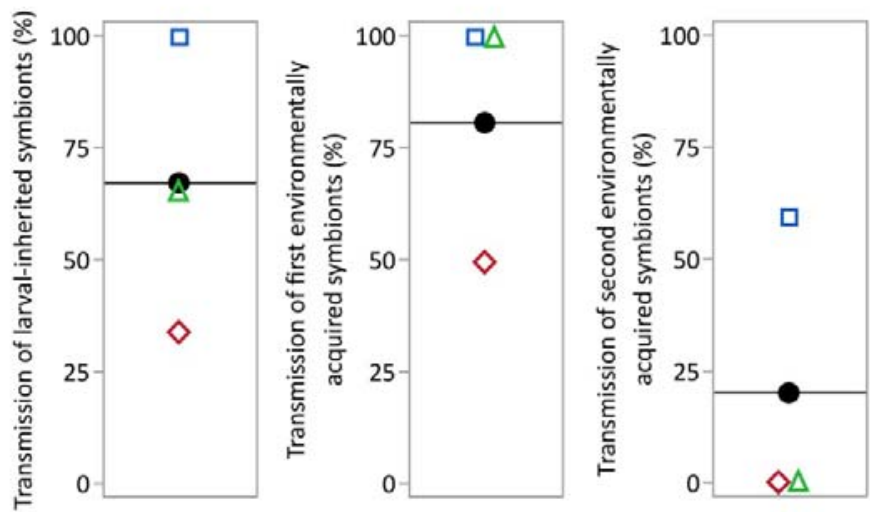

Figure 3. Larval yeast symbionts maintain through Drosophila stages and generations. (A) Experimental design. $\mu$ means microbial community. (B) Maintenance of symbionts through metamorphosis. (C) Maintenance of larval symbionts and acquisition of environmental symbionts in adults. (D) Transmission of the different adult symbionts to a new fly generation. The black dot symbolizes the general mean (i.e. independently of the microbial symbiont). 


\section{Conclusion}

195 We discovered that Drosophila females and males both transmit their extracellular symbionts 196 to larvae. Several symbiotic yeasts initially associated with larvae were conserved throughout 197 host life cycle and transmitted to offspring. Our results, mainly obtained with microorganisms freshly isolated in the wild, suggest that stable associations of Drosophila flies with bacteria and yeasts may exist in natura. As our results were obtained under ecologically realistic conditions, they may therefore constitute a tangible step forward in the understanding of wild Drosophila - microorganism symbioses.

A major issue in the recent Drosophila literature is to determine how exactly microbial symbionts maintain association with the host. Most studies conclude Drosophila microbial symbionts do not maintain durably in the host. Symbionts would be continuously inoculated by the host to the substrate where they multiply, reacquired from the environment via a 'farming' mechanism but rarely conserved in absence of intake during feeding. However, this phenomenon has been described using laboratory strains of Drosophila and symbionts under typical laboratory conditions (Blum et al. 2013; Storelli et al. 2018). Along these lines, several studies show extracellular symbionts found in arthropods reflect the microbial communities they encounter in their diet (Kennedy et al. 2020; Moran et al. 2019). By contrast, evidence of the existence of resident extracellular symbionts of Drosophila accumulates. In D. melanogaster adults, two recent independent studies show that wild isolates of the bacteria Lactobacillus plantarum and Acetobacter thailandicus may durably colonize the first gut region of the host (crop, crop duct and proventriculus) independently of the ingestion of other symbionts under laboratory conditions (Pais et al. 2018; Obadia et al. 2017). In the wild, such resident symbionts may durably persist in host individuals and populations. Our study was not designed to investigate how and why extracellular Drosophila symbionts persist in or get lost by adult hosts. However, we found symbiont maintain throughout metamorphosis, a phenomenon that was poorly studied with wild strains in fruit (Bakula 1969; Ridley et al. 2012; Starmer et al. 1988). Differences among yeasts strains in terms of maintenance and transmission may relate to where they locate in the host and therefore how we sampled them. Indeed, the yeast Trigonopsis vinaria we isolated from Drosophila ovaries best maintained throughout metamorphosis (Figure 4B). However, Hanseniaspora uvarum, a species frequent on the surface of fruit (Morais et al. 1995), that 
225 strongly attracts Drosophila adults and is often found associated with them, always 226 transmitted well from adults to larvae (Figures 2 and 3). If it is not possible to generalize with 227 a handful of microbial strains, the data suggests wild symbionts vary in their strategies of 228 host-mediated dispersal (Jacob et al. 2019). Most yeast species rely on insect vectors for 229 dispersal (Kurtzman et al. 2011), some may be better at attracting adults, others at 230 transmitting among life stages or to offspring. Recent literature debates whether yeast 231 coevolve with flies on the basis that the volatiles they produce have other functions than just 232 to attract flies (Günther et al. 2019; Koerte et al. 2020). The contingency of each other's 233 fitness due to yeast maintenance during metamorphosis and transmission from adults to larvae 234 constitutes another coevolutionary paradigm. One where symbiotic associations are not solely 235 driven by partner choice but also by co-transmission (Sachs et al. 2004). When host and 236 symbiont fitnesses correlate positively selection favors mutualistic interactions (Ebert 2013; 237 Lipsitch et al. 1996; Sachs et al. 2004). In Drosophila, benevolent effects of extracellular 238 symbionts may amount to better provisioning of nutriments (Ankrah \& Douglas 2018) or host 239 protection against pathogens (Johnston \& Rolff 2015). Future research will tell whether yeast 240 - and symbiotic bacteria - harbor adaptations favoring long-term associations with hosts and 241 maximize their own fitness by mutualistic influence on their host.

242 Symbiont persistence has broad consequences for the eco-evolutionary dynamics of host and 243 symbionts in heterogeneous environments. The maintenance of symbionts over days or 244 generations enables their participation to host adaptation to local conditions. In return 245 benevolent symbionts may benefit improved dispersal to new resource patches. For that 246 matter, orchards, shrubs and cities where Drosophila and their symbionts may be encountered 247 resembles the very definition of meta-populations: fruits are ephemeral patches of finite 248 resources from which it is necessary to disperse to survive in the long run. Incidentally, 249 understanding how hosts acquire and transmit non-obligatory symbionts, such as the bacteria 250 and yeast we studied here, helps with a major challenge for the years to come. The ecological 251 and evolutionary dynamics of most microorganisms in space and time remains obscure, in 252 particular in structured, complex environments (Dudaniec \& Tesson 2016). Empirical study of 253 opportunistic symbionts in natural conditions or with field-realistic microcosms will shed 254 light on some of this mystery. 


\section{Drosophila stocks and microbial symbionts}

257

258

259

260

261

262

263

264

265

266

267

268

We used two populations of Drosophila melanogaster (Population A, founded from OregonR individuals furnished by colleagues, and Population B, founded from wild individuals collected in the late 2017 around Montpellier, Southern France) and two populations of $D$. suzukii (Population A, founded from wild individuals collected in the early 2018 around Avignon, Southern France, and Population B, founded from wild individuals collected in 2013 in Gaujac, Southern France).

We used six microbial symbionts in this study. Five were isolated from wild flies and fruits in the late 2017. The yeasts Hanseniaspora uvarum MN684824, Trigonopsis vinaria MN684816 and Rhodotorula babjevae MN684819 were isolated from female D. melanogaster feces, D. suzukii ovaries and infested organic grape berries, respectively. The bacteria Serratia liquefaciens and Gluconobacter thailandicus were respectively isolated from D. suzukii ovaries and organic grape berries. The sixth microorganism was a laboratory isolate of the bacterium Lactobacillus plantarum which is widely used in bacteria - Drosophila studies (Ryu et al. 2008). Colonies of these six isolates were distinguished according to their morphology (e.g. Figure 4). More details about these isolates (their choice, their properties, the method to distinguish them) will be given in the next version of this work.

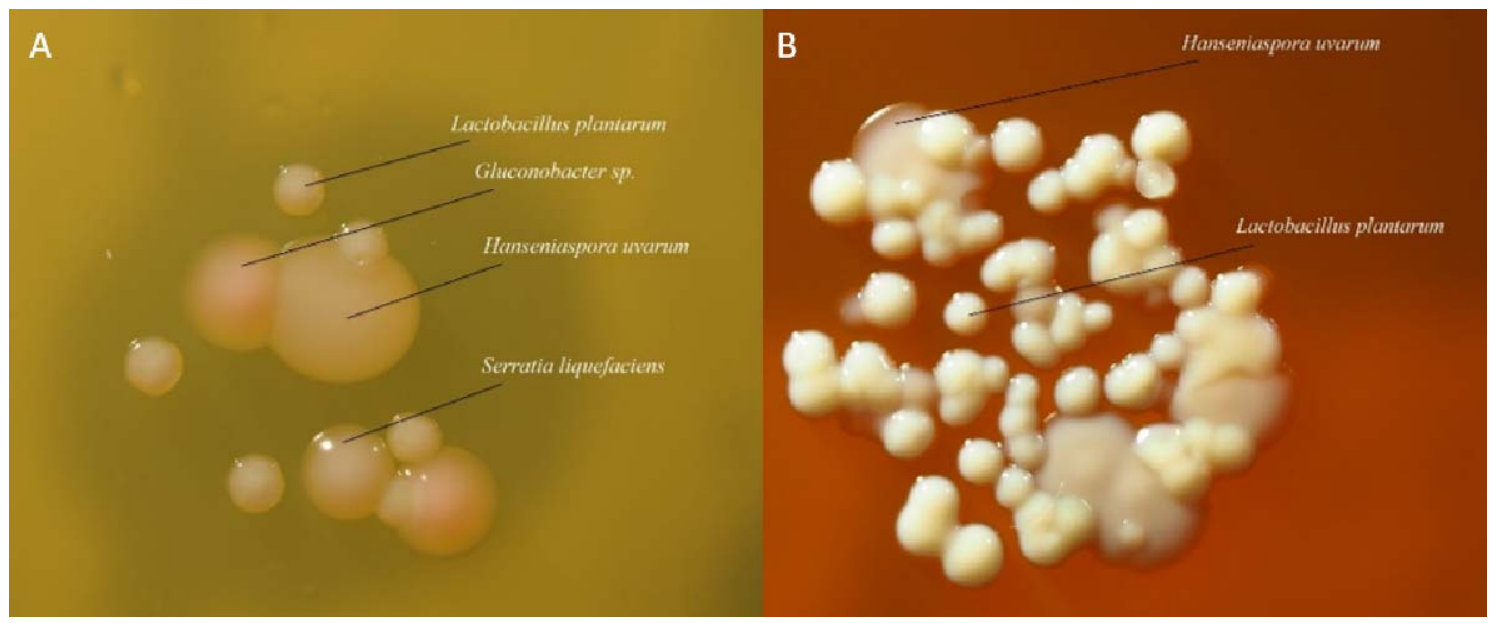

Figure 4. Colony morphology as a tool for discriminating mixed microbial isolates. (A) MRS agar plate (incubated at $30^{\circ} \mathrm{C}$ ) allowed to distinguish colonies of four microbial isolates. (B) Mannitol agar plate (incubated at $24^{\circ} \mathrm{C}$ ) allowed to distinguish colonies of two microbial isolates. 


\section{Origin of larval microbiota}

280 The experiments were conducted on sterile vials with conventional blueberries and 281 gnotobiotic Drosophila adults, i.e. associated with particular microbial symbionts.

282 Gnotobiotic adults were created by inoculating axenic larvae or adults (i.e. free of extracellular symbionts here) with overnight grown microbial symbionts (MRS $30^{\circ} \mathrm{C}$ for $L$. plantarum, Mannitol $24^{\circ} \mathrm{C}$ for other bacteria, YPD $24^{\circ} \mathrm{C}$ for yeasts). The axenic colonies of Drosophila were founded with axenic eggs obtained from conventionally reared populations using a method slightly adapted from Koyle and colleagues (2016). Briefly, this method consists of removing the chorion, the outer envelope of the egg that contains extracellular microbial symbionts. The axenic colonies were maintained on sterile banana medium (water, banana, sugar, dead yeast and agar).

Blueberries were always disposed with peduncle insertion upwards. This particular zone of the berry was identified as a preferential oviposition site for D. suzukii females (Figure 5). To allow oviposition of $D$. melanogaster females, this zone was finely wounded using a pipette tip. All blueberries used in the experiments were surface-sterilized following the protocol of Behar and colleagues (2008). For the two main experiments of this section, surface-sterilized blueberries were artificially associated with microbial symbionts. To this aim, berries were immersed in microbial suspensions (overnight microbial culture diluted in PBS (Phosphate Buffered Saline)) and dried $18 \mathrm{~h}$ after a 2 min 30 vortexing.

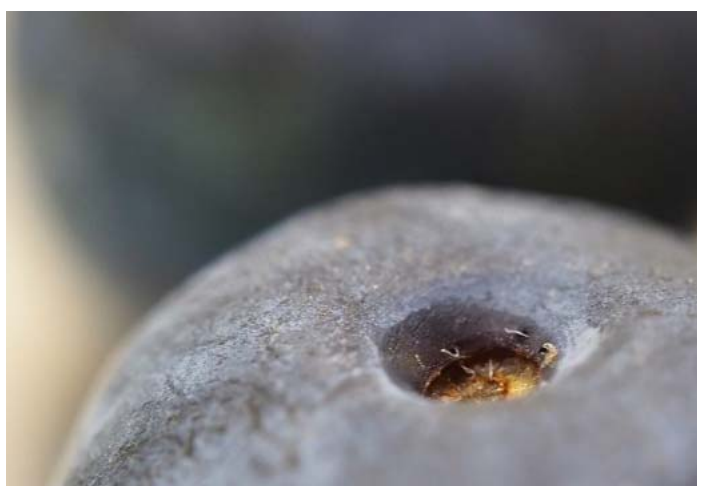

Figure 5. D. suzukii eggs are laid around the insertion of the fruit peduncle.

$\underline{\text { Maternal transmission }}$

We used D. melanogaster population B, D. suzukii population B and the six microbial symbionts. Females were reared with males and associated with microbial symbionts five 
304

305

306

307

308

309

310

311

312

313

314

315

316

317

318

319

320

321

322

323

324

325

326

327

328

329

330

331

332

333

334

335

336

days before the experiment. Each experimental unit was constituted of one mature female and a wounded (D. melanogaster) or intact (D. suzukii) blueberry. Female and fruit were associated with a different microbial community (i.e. a different bacterium and a different yeast). For this experiment only, blueberries were inoculated with two different concentrations of microbial symbionts: a low concentration (5000 cells per microbial symbiont, decided in the light of previous estimates of cell numbers deposited by insects on fruit surfaces) and a high concentration (50 000 cells per microbial symbiont). Our initial goal was to test whether the concentration of fruit-associated microbial symbionts influences their transmission to the larvae, that was not the case (Table S1). A wet sterile cotton piece was added into each vial to ensure adult hydration. We created 36 female-fruit microbial combinations $*$ two concentrations $=72$ vials per Drosophila species. Females were disposed on fruits at $5 \mathrm{pm}$ for $24 \mathrm{~h}$. Controls without females were created to detect potential exogenous extracellular microorganisms. Adults were collected at the end of the day, crushed in PBS $+20 \%$ glycerol and stocked at $-80^{\circ} \mathrm{C}$. After five days, up to ten larvae were collected per fruit, pooled and crushed in PBS using a Tissue Lyser II. Right after crushing, larval samples were simultaneously plated on Galactose, Glucose, Mannitol (incubation at $24^{\circ} \mathrm{C}$ ) and MRS plates (incubation at $30^{\circ} \mathrm{C}$ ) to differentiate between and count microbial symbionts.

\section{Male-mediated transmission}

We used D. melanogaster population B, D. suzukii population B and the six microbial symbionts. The used adults were obtained from larvae associated with a combination of one yeast and one bacterium. Emerging male and female adults were kept five days in the same vials then separated per sex and re-associated with the original yeast + bacterium combination. Each experimental unit was constituted of one mature female, one mature male and a wounded (D. melanogaster) or intact (D. suzukii) blueberry. Prior to the experiment, each female, male and fruit were associated with a different microbial community (i.e. a different bacterium and a different yeast). For this experiment, blueberries were inoculated with 5000 microbial cells of each symbiont. A wet sterile cotton piece was added into each vial to ensure adult hydration. Per Drosophila species, we created 36 vials, one for each malefemale-fruit microbial combination. In the early morning, the male was placed on the vial to enable the deposition of its microbial symbionts on the fruit surface. Note we verified the capability of males to deposit their symbionts on the fruit surface during a preliminary essay, this data will be presented in the next version of this work. To encourage the male to sit on the 
337 fruit, an axenic mature female kept in a small cage was added to the system (Figure 6). Male

338 presence on the oviposition site was recorded eight times along the day. In the early morning 339 of a second day, the captive axenic female was removed from the vial and the free-living 340 gnotobiotic female was added. Male presence on the oviposition site was recorded eight times 341 along this second day. Mating was also recorded every $30 \mathrm{~min}$. Controls without females and 342 males were created to detect potential exogenous extracellular microorganisms. Individuals 343 were collected at the end of the second day, crushed in PBS $+20 \%$ glycerol and stocked at $34480^{\circ} \mathrm{C}$. After five days, up to ten larvae were collected per fruit, pooled and crushed in PBS 345 using a Tissue Lyser II. Right after crushing, larval samples were simultaneously plated on 346 Galactose, Glucose, Mannitol (incubation at $24^{\circ} \mathrm{C}$ ) and MRS plates (incubation at $30^{\circ} \mathrm{C}$ ) to 347 differentiate between and count microbial symbionts.

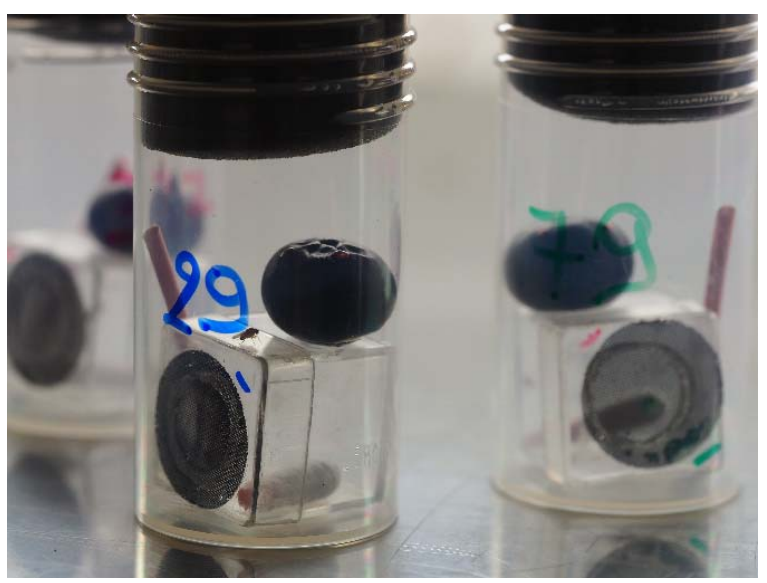

Figure 6. Example of experimental units used to test male transmission (on the first day, with a free-living gnotobiotic male and an axenic female in cage).

Maintenance and transmission of microbial symbionts throughout the insect life cycle and between generations

We used D. melanogaster population B, D. suzukii population A and the three yeast isolates. Grape juice plates supplemented with the antifungal cycloheximide $(1 \mu \mathrm{l} / 10 \mathrm{ml})$ were used to obtain yeast-free eggs from conventionally reared females. Eggs were deposited on surfacesterilized, incised grape berries (Behar et al., 2008) disposed on sterile vermiculite. After egg deposition, the wounds were inoculated with a single yeast strain (from overnight culture in YPD at $\left.24^{\circ} \mathrm{C}\right)$. After the end of pupal formation, fruits were removed. Five freshly emerged adults of each sex were collected to evaluate yeast persistence through host metamorphosis. Other adults were placed in new experimental units. Each experimental unit was constituted 
362

363

364

365

366

367

368

369

370

371

372

373

374

375

376

377

378

379

380

381

382

383

384

385

386

387

388

389

390

of one male and one female that were reared with the same yeast strain. A petri dish with a wet cotton piece and sugar was disposed in the system to ensure fly survival. Right after setting of the system, a first grape berry inoculated with a second yeast strain was added. After five days, the fruit was removed and a second grape berry inoculated with a third yeast strain was added. Two days after, the fruit was removed and an incised, surface-sterilized grape berry was added to collect larvae. After one day, the adults were collected. Three days after, larvae were aseptically removed from fruit flesh. All adult and larval samples were crushed in PBS right after their collect using a Tissue Lyser II (Qiagen) and plated on Galactose and Glucose plates to differentiate and count yeast symbionts.

\section{Statistical analyses}

GLM models with binomial distribution and logit function or poisson distribution and log function were used using JMP (SAS, 14.1). A backward stepwise model selection was used to eliminate non-significant terms from initial full models.

\section{Acknowledgements}

We thank Laure Benoit, Marie-Pierre Chapuis and Romain Gallet for their help for the molecular identification of the microbial isolates used in this study and during the preliminary experiments.

\section{References}

Ankrah, N. Y. D., \& Douglas, A. E. (2018). Nutrient factories: Metabolic function of beneficial microorganisms associated with insects. Environmental Microbiology, 00, 1-10.

Antonovics, J., Wilson, A. J., Forbes, M. R., Hauffe, H. C., Kallio, E. R., Leggett, H. C., Longdon, B., Okamura, B., Sait, S. M., \& Webster, J. P. (2017). The evolution of transmission mode. Philosophical Transactions of the Royal Society B: Biological Sciences, 372(1719), 20160083.

Bakula, M. (1969). The persistence of a microbial flora during postembryogenesis of Drosophila melanogaster. Journal of Invertebrate Pathology, 14(3), 365-374. 
391 Becher, P. G., Flick, G., Rozpedowska, E., Schmidt, A., Hagman, A., Lebreton, S., Larsson,

392 M. C., Hansson, B. S., Piškur, J., Witzgall, P., \& Bengtsson, M. (2012). Yeast, not fruit volatiles mediate Drosophila melanogaster attraction, oviposition and development. Functional Ecology, 26(4), 822-828.

Behar, A., Jurkevitch, E., \& Yuval, B. (2008). Bringing back the fruit into fruit fly-bacteria interactions. Molecular Ecology, 17(5), 1375-1386.

Blum, J. E., Fischer, C. N., Miles, J., \& Handelsman, J. (2013). Frequent replenishment sustains the beneficial microbiome of Drosophila melanogaster. MBio, 4(6).

Bright, M., \& Bulgheresi, S. (2010). A complex journey: transmission of microbial symbionts. Nature Reviews Microbiology, 8(3), 218.

401 Drapeau, M. D., Fuller, B. F., Rauser, C. L., \& Long, A. D. (2011). Repeated mating in a lekmating insect, Drosophila melanogaster. Dros. Inf. Serv., 84, 136-140.

Dudaniec, R. Y., \& Tesson, S. V. M. (2016). Applying landscape genetics to the microbial world. Molecular ecology, 25(14), 3266-3275.

Duneau, D. F., \& Lazzaro, B. P. (2018). Persistence of an extracellular systemic infection across metamorphosis in a holometabolous insect. Biology Letters, 8-11.

Ebert, D. (2013). The epidemiology and evolution of symbionts with mixed-mode transmission. Annual Review of Ecology, Evolution, and Systematics, 44, 623-643.

Fisher, R., Henry, L., Cornwallis, C., Kiers, E., \& West, S. (2017). The evolution of hostsymbiont dependence. Nature Communications, 8, 15973.

411 Funkhouser, L. J., \& Bordenstein, S. R. (2013). Mom Knows Best: The Universality of Maternal Microbial Transmission. PLoS Biology, 11(8), 1-9. evolutionary ecology of symbioses between host and microbes. BMC Biology, 15(1), 4-9.

415 Gonella, E., Crotti, E., Rizzi, A., Mandrioli, M., Favia, G., Daffonchio, D., \& Alma, A. 416 (2012). Horizontal transmission of the symbiotic bacterium Asaia sp. in the leafhopper 417 Scaphoideus titanus Ball (Hemiptera: Cicadellidae). BMC Microbiology, 12(Supp1), 1-13.

418 Günther, C. S., Knight, S. J., Jones, R., \& Goddard, M. R. (2019). Are Drosophila preferences 419 for yeasts stable or contextual? Ecology and Evolution, 9(14), 8075-8086. 
420 Hoffmann, A. (1987). A laboratory study of male territoriality in the sibling species

421 Drosophila melanogaster and Drosophila simulans. Animal Behaviour, 35, 807-818.

422 Hoffmann, A., \& Cacoyianni, Z. (1990). Territoriality in Drosophila melanogaster as a 423 conditional strategy. Animal Behaviour, 40(3), 526-537.

424 Jacob, S., Chaine, A. S., Huet, M., Clobert, J., \& Legrand, D. (2019). Variability in Dispersal 425 Syndromes Is a Key Driver of Metapopulation Dynamics in Experimental Microcosms. The 426 American Naturalist, 194(5), 613-626.

427 Johnston, P. R., \& Rolff, J. (2015). Host and Symbiont Jointly Control Gut Microbiota during 428 Complete Metamorphosis. PLoS Pathogens, 11(11).

429 Kennedy, S. R., Tsau, S., Gillespie, R., \& Krehenwinkel, H. (2020). Are you what you eat? A 430 highly transient and prey $\square$ influenced gut microbiome in the grey house spider Badumna 431 longinqua. Molecular Ecology, 29(5), 1001-1015.

432 Kikuchi, Y., Hosokawa, T., \& Fukatsu, T. (2007). Insect-Microbe Mutualism without Vertical 433 Transmission: a Stinkbug Acquires a Beneficial Gut Symbiont from the Environment Every 434 Generation. Appl. Environ. Microbiol., 73(13), 4308-4316.

435 Koerte, S., Keesey, I. W., Easson, M. L. A. E., Gershenzon, J., Hansson, B. S., \& Knaden, M. 436 (2020). Variable dependency on associated yeast communities influences host range in 437 Drosophila species. Oikos, oik.07180. Koyle, M. L., Veloz, M., Judd, A. M., Wong, A. C. N., Newell, P. D., Douglas, A. E., \& Chaston, J. M. (2016). Rearing the Fruit Fly Drosophila melanogaster Under Axenic and Gnotobiotic Conditions. Journal of Visualized Experiments $\square:$ JoVE, 113, e54219.

441 Kurtzman, C. P., Fell, J. W., \& Boekhout, T. (2011). The Yeasts - Fifth edition.

442 Lewis, M., \& Hamby, K. (2019). Differential impacts of yeasts on feeding behavior and 443 development in larval Drosophila suzukii (Diptera: Drosophilidae). Scientific Reports, 9(1), $444 \quad 1-12$.

445 Lipsitch, M., Nowak, M. A., \& Siller, S. (1996). The evolution of virulence in pathogens with 446 vertical and horizontal transmission. Evolution, 50(5), 1729-1741.

447 Ma, D., \& Leulier, F. (2018). The importance of being persistent: The first true resident gut 448 symbiont in Drosophila. PLOS Biology, 16(8), e2006945. 
Miest, T. S., \& Bloch-Qazi, M. C. (2008). Sick of mating sexual transmission of a pathogenic bacterium in Drosophila melanogaster. Fly, 2(4), 215-219.

Morais, P. B., Martins, M. B., Klaczko, L. B., Mendonca-Hagler, L. C., \& Hagler, A. N. (1995). Yeast succession in the amazon fruit Parahancornia amapa as resource partitioning among Drosophila spp. Applied and Environmental Microbiology, 61(12), 4251-4257.

Moran, N. A., Ochman, H., \& Hammer, T. J. (2019). Evolutionary and Ecological

455 Consequences of Gut Microbial Communities. Annual Review of Ecology, Evolution, and 456 Systematics, 50(1), 451-475. Current Biology, 27(13), 1999-2006.

Pais, I. S., Valente, R. S., Sporniak, M., \& Teixeira, L. (2018). Drosophila melanogaster establishes a species-specific mutualistic interaction with stable gut-colonizing bacteria. PLoS Biology, 16(7).

Quigley, K. M., Warner, P. A., Bay, L. K., \& Willis, B. L. (2018). Unexpected mixed-mode transmission and moderate genetic regulation of Symbiodinium communities in a brooding coral. Heredity, 121, 524-536.

466 Reaume, C. J., \& Sokolowski, M. B. (2006). The nature of Drosophila melanogaster. Current Biology, 16(16), 623-628.

Ridley, E. V., Wong, A. C. N., Westmiller, S., \& Douglas, A. E. (2012). Impact of the resident microbiota on the nutritional phenotype of Drosophila melanogaster. PLoS ONE, $7(5)$.

471 Rohlfs, M., \& Hoffmeister, T. S. (2005). Maternal effects increase survival probability in Drosophila subobscura larvae. Entomologia Experimentalis et Applicata, 117(1), 51-58.

473 Sachs, J. L., Mueller, U. G., Wilcox, T. P., \& Bull, J. J. (2004). The evolution of cooperation. 474 The Quarterly Review of Biology, 79(2), 135-160.

475 Shapiro, J. W., \& Turner, P. E. (2014). The impact of transmission mode on the evolution of 476 benefits provided by microbial symbionts. Ecology and Evolution, 4(17), 3350-3361. 
477 Spencer, D. M., Spencer, J. F. T., De Figueroa, L., \& Heluane, H. (1992). Yeasts associated 478 with rotting citrus fruits in Tucumán, Argentina. Mycological Research, 96(10), 891-892.

479 Starmer, W. T., Peris, F., \& Fontdevila, A. (1988). The transmission of yeasts by Drosophila 480 buzzatii during courtship and mating. Animal Behaviour, 36(6), 1691-1695.

481 Stefanini, I., Dapporto, L., Legras, J. L., Calabretta, A., Di Paola, M., De Filippo, C., Viola, 482 R., Capretti, P., Polsinelli, M., Turillazzi, S., \& Cavalieri, D. (2012). Role of social wasps in 483 Saccharomyces cerevisiae ecology and evolution. Proceedings of the National Academy of 484 Sciences of the United States of America, 109(33), 13398-13403.

485 Storelli, G., Strigini, M., Grenier, T., Bozonnet, L., Schwarzer, M., Daniel, C., Matos, R., \& 486 Leulier, F. (2018). Drosophila Perpetuates Nutritional Mutualism by Promoting the Fitness of 487 Its Intestinal Symbiont Lactobacillus plantarum. Cell Metabolism, 27(2), 362-377.

488 Taylor, C. E., \& Kekic, V. (1988). Sexual Selection in a Natural Population of Drosophila 489 melanogaster. Evolution, 42(1), 197.

490 Téfit, M. A., Gillet, B., Joncour, P., Hughes, S., \& Leulier, F. (2018). Stable association of a 491 Drosophila-derived microbiota with its animal partner and the nutritional environment 492 throughout a fly population's life cycle. Journal of Insect Physiology, 106, 2-12.

493 Woltz, J. M., \& Lee, J. C. (2017). Pupation behavior and larval and pupal biocontrol of 494 Drosophila suzukii in the field. Biological Control, 110, 62-69. 